\title{
CTC Regimen
}

National Cancer Institute

\section{Source}

National Cancer Institute. CTC Regimen. NCI Thesaurus. Code C9685.

A chemotherapy regimen consisting of cyclophosphamide, thiotepa, and carboplatin that may be used in the treatment of breast cancer. 